\title{
Clinical study on different doses and fractionated radiotherapies for multiple brain metastases of non-EGFR mutant lung adenocarcinoma
}

\author{
Hongli Liu ${ }^{1,2 \#}$, Xuedong $\mathrm{Xu}^{2 \#}$, Jinzhi Wang ${ }^{2}$, Wei Wang ${ }^{2}$, Changsheng $\mathrm{Ma}^{3}$, Tao Sun ${ }^{3}$, Qian Shao ${ }^{2}$ \\ ${ }^{1}$ School of Medicine and Life Science, University of Jinan-Shandong Academy of Medical Sciences, Jining Tumor Hospital, Jinan, China; \\ ${ }^{2}$ Department of Radiation Oncology (Chest Section), ${ }^{3}$ Department of Radiophysics and Technology, Shandong Cancer Hospital and Institute, \\ Shandong First Medical University and Shandong Academy of Medical Sciences, Jinan, China \\ Contributions: (I) Conception and design: Q Shao; (II) Administrative support: Q Shao; (III) Provision of study materials or patients: H Liu, X Xu; \\ (IV) Collection and assembly of data: J Wang, W Wang, C Ma, T Sun; (V) Data analysis and interpretation: H Liu, X Xu, Q Shao; (VI) Manuscript \\ writing: All authors; (VII) Final approval of manuscript: All authors. \\ \#The authors contributed equally to this work. \\ Correspondence to: Qian Shao. Department of Radiation Oncology (Chest Section), Shandong Cancer Hospital and Institute, Shandong First Medical \\ University and Shandong Academy of Medical Sciences, Jinan 250117, China. Email: shaoqian2009@sina.com.
}

Background: To study the effects and adverse reactions of different doses and fractionated radiotherapies on non-epidermal growth factor receptor (EGFR) mutant lung adenocarcinoma patients with multiple brain metastases.

Methods: In total, 80 patients eligible for inclusion were randomly divided into 4 groups. Group A included whole brain radiotherapy (WBRT) alone $300 \mathrm{cGy} /$ fraction $\times 10$ fractions, at a total dose of 3,000 cGy. Group B included WBRT alone $180 \mathrm{cGy} /$ fraction $\times 22$ fractions, at a total dose of 3,960 cGy. Group C included intracranial metastases radiotherapy alone $250 \mathrm{cGy} /$ fraction $\times 22$ fractions at a total dose of 5,500 cGy. Group D included the whole brain + intracranial metastases group (SIB group) whole brain 180 cGy/fraction $\times 22$ fractions intracranial metastases $250 \mathrm{cGy} /$ fraction $\times 22$ fractions, at a total dose 3,960 cGy, 5,500 cGy, respectively.

Results: The median survival time of intracranial disease-free survival (IPFS) in group A, group B, group $\mathrm{C}$, and group $\mathrm{D}$ was $6,9,8$, and 13 months, respectively $(\mathrm{P}=0.000)$. The median overall survival (OS) time was $16,24.5,24$, and 30 months, respectively $(\mathrm{P}=0.150)$. There was a significant difference in IPFS between different doses and fractionated radiotherapies, but there was no difference in OS. Multivariate analysis showed that the radiotherapy dose of intracranial metastases was positively correlated with IPFS and OS. The incidence rate of adverse reaction of memory decline in $0.5,1$, and 2 years in group A, group B, group C, and group D was respectively $10.0 \%, 15.0 \%, 5.0 \%$, and $15.0 \%(\mathrm{P}=0.006) ; 20.0 \%, 45.0 \%, 30.0 \%$, and $60.0 \%(\mathrm{P}=0.000) ; 10.0 \%, 20.0 \%, 35.0 \%$, and $65.0 \%(\mathrm{P}=0.000)$. The incidence rates of memory decline in the groups of WBRT were significantly more increased than in the non-WBRT group.

Conclusions: Radiotherapy is effective for multiple brain metastases of lung adenocarcinoma, the increase of radiotherapy dose can improve IPFS and OS, and the adverse reaction of memory decline after WBRT is increased but tolerable. Therefore, WBRT and simultaneous integrated boost (SIB) radiotherapy of intracranial metastases is recommended for multiple brain metastases of non-EGFR-mutant lung adenocarcinoma.

Keywords: Lung adenocarcinoma; brain metastases; non-EGFR-mutant; radiotherapy; dose; survival time

Submitted Apr 09, 2020. Accepted for publication Jul 17, 2020.

doi: 10.21037/apm-20-1203

View this article at: http://dx.doi.org/10.21037/apm-20-1203 


\section{Introduction}

The brain is the most common metastatic organ of lung adenocarcinoma in which multiple brain metastases (number of metastatic sites $\geq 3$ ) are more likely be discovered. Radiotherapy is an important treatment for patients with multiple brain metastases, yet, the doses and fractionated radiotherapies have not standardized. Lung cancer is the most common malignant tumor worldwide, and its morbidity and mortality are the first among the malignant tumors. Approximately $10 \%$ of patients at the initial diagnosis of non-small cell lung cancer (NSCLC) have combined brain metastasis, and those without brain metastasis at the beginning treatment will eventually develop brain metastasis during the whole course of the disease. Across difference cancer types, approximately $70 \%$ of cases have combined multiple brain metastases. Because of the existence of the blood-brain barrier, the effectiveness of chemotherapy on brain metastasis is rather weak. As for asymptomatic lung adenocarcinoma patients with the epidermal growth factor receptor (EGFR) mutation, targeted therapy can be used as the initial treatment option, while surgery and radiotherapy are the effective treatment options for patients with symptomatic brain metastases or non-EGFR mutant lung adenocarcinoma with isolated brain metastasis. The therapy of patients with multiple brain metastases is a palliative treatment, and the chance of selecting surgery is rare. At present, bevacizumab (a targeted drug against angiogenesis) significantly reduces the incidence of brain metastasis and immunotherapy can improve the survival time of patients with brain metastasis in lung cancer. In addition, as a local treatment, radiotherapy has become the primary treatment for patients with multiple brain metastases, especially for those lung adenocarcinoma patients with non-EGFR mutant multiple brain metastases. Radiotherapy has the advantages of being non-invasive, economic, effective, safe, and reliable. However, there is no clinical standard for the target radiotherapy and the dose of fractionated radiotherapy. Therefore, it is urgent to clarify the target, fractionated times and dose of the radiotherapy.

The brain is the most common malignant tumor metastatic site, and about $25-40 \%$ of extracranial malignant brain metastases occur in the course of lung cancer. Common primary tumors such as lung cancer, breast cancer, digestive tract cancer, kidney cancer, lung cancer account for $60 \%$ to $70 \%$ of brain metastases. As state above, most NSCLC patients will eventually acquire brain metastases, with the highest incidence of brain metastases occurring in lung adenocarcinoma patients. Therefore, discussing the cost, efficacy, safety, and reliability of radiotherapy is an urgent clinical issue. For single brain metastasis with a Karnofsky performance score $0-2$ and a good prognosis, surgery or stereotactic radiosurgery (SRS) is the first choice. Retrospective study data suggest that SRS and surgery have similar efficacy in patients with a single brain metastasis. Compared with whole brain radiotherapy (WBRT), SRS improves the median survival of patients with 1-3 brain metastases, and so SRS is the preferred treatment for these patients.

Radiotherapy for patients with multiple brain metastases has evolved from WBRT or optimal supportive therapy from before the 1980s to the current era when stereotactic and intensity-modulated radiotherapy have become widely used. Most retrospective research suggests that although stereotactic radiotherapy combined with WBRT does not improve the total survival of patients when compared to WBRT alone, it can significantly improve the intracranial local control rate of patients and the quality of life of those patients. In recent years, with the growing attention paid to the cognitive function of patients with brain metastatic tumor, it is advised that patients with brain metastatic tumor with a metastatic site be treated with focused radiotherapy instead of WBRT. Randomized studies have examined the association between cognitive function and the influence of survival with the choice of stereotactic radiotherapy or stereotactic radiotherapy in combination with WBRT. The results showed that without whole brain radiation therapy, intracranial control was significantly reduced, and salvage therapy did not affect the overall survival (OS) of patients. As for the influence on cognitive function in patients with brain metastases, these results were not consistent, and require further research. In conclusion, the current radiotherapy for multiple brain metastases still lacks a unified standard treatment model.

With the development of imaging technology and indepth research, the location of brain metastatic tumors has become more accurate, which provides a possibility for the implementation of simultaneous integrated boost (SIB) radiotherapy for whole brain and intracranial metastatic sites. As SIB can also improve the local control rate and reduce the occurrence of recurrence, it may be ideal as a standard for radiotherapy in multiple brain metastases.

We present the following article in accordance with the CONSORT reporting checklist (available at http://dx.doi. org/10.21037/apm-20-1203). 


\section{Research background}

Patients with lung cancer with brain metastasis who are not treated or who receive only symptomatic treatment, have a median survival of 1-2 months (1), but survival time can significantly be improved with effective treatment $(2,3)$.

For multiple brain metastases, radiotherapy is the most common and most effective treatment method, and WBRT has been the standard treatment of multiple brain metastases, but it has certain adverse effects on the patient's quality of life.

Radiotherapy of metastatic brain tumor combined with WBRT can increase the doses to the metastatic brain tumor and shorten the treatment time. However, further studies are needed to determine whether this treatment mode is helpful in improving the survival of patients. Therefore, in order to identify a standard for the above parameters, we conducted a prospective study in multiple brain metastases patients at initial treatment.

\section{Methods}

\section{General clinical data}

Participants in this study included lung adenocarcinoma patients who were first treated in Shandong tumor Hospital Radiation Ward from June 1, 2015 to June 31, 2017. The inclusion criteria for patients were the following: lung adenocarcinoma confirmed by bronchoscopy or lung biopsy; non-EGFR-mutated and non-anaplastic lymphoma kinase (ALK)-mutated as confirmed by genetic testing; presence of 5-9 brain metastases according to imaging, with or without other distant metastasis; no history of brain radiotherapy or surgery; no previous history of tumor in other organs and no major organ dysfunction; normal for blood routine, liver and kidney function, electrolyte, blood glucose, electrocardiogram, and other standard measures; an Eastern Cooperative Oncology Group (ECOG) score $0-2$. The study was conducted in accordance with the Declaration of Helsinki (as revised in 2013). Approval was obtained from the ethics committee of our hospital (No. SDTHEC201503048), and the patients signed the informed consent and were followed up regularly as required.

A total of 80 patients were enrolled in this study and randomly divided into 4 group, with 20 patients in each group. Group A was treated with whole-brain radiotherapy alone. The dose and fractionated radiotherapy were 3,000 cGy/10 factions, and the total amount was 3,000 cGy. Group B was the second WBRT. The target volume of radiotherapy was the whole brain, the dose and fractionated radiotherapy was $180 \mathrm{cGy} / 22$ factions, and the total amount was 3,960 cGy. Group C was the radiotherapy group with intracranial metastasis alone; the radiotherapy target was the intracranial metastasis site, and the dose and fractionated radiotherapy was $250 \mathrm{cGy} / 22$ factions, with a total amount of 5,500 cGy. Group D was the whole brain + intracranial metastasis group; The dose for fractionated radiotherapy was $180 \mathrm{cGy} / 22$ fractions for the total brain, the dose for fractionated radiotherapy was $250 \mathrm{cGy} / 22$ fractions for the intracranial metastases, the total brain amount was 3,960 cGy, and the total metastasis amount was 5,500 cGy.

Among the 80 patients, 45 were male and 35 were female. Ages ranged from 33 to 77 years, the mean age was $56.33 \pm 9.97$ years, and the median age was 57.5 years. The number of brain metastases ranged from 5 to 9, with an average of $5.99 \pm 1.119$ and a median of 6 . The 4 groups' general information, single-factor variance $\left(\mathrm{X}^{2}\right)$ inspection (count) and t measurement showed no difference (Table 1).

Patients were tested for memory decline using the Simple Mini-Mental State Examination (MMSE).

According to the different enrollment time, an informed agreement is signed, and the patients are randomly divided into groups, and the corresponding radiotherapy plan is done for radiotherapy.

\section{Localization of the brain with computed tomography and magnetic resonance imaging}

The patient was placed in supine position on the head fixation frame, and a thermoplastic mask was fixed. A 3 -point marking method was adopted on the brow arch level of the mask surface to facilitate the repeatability and accuracy of head fixation and positioning, and to determine the reference points and isocentric points during planning. Philips Brilliance wide-aperture enhanced computed tomography (CT) scan with $3 \mathrm{~mm}$ lamellar thickness was used for continuous scanning, and the same method was used for enhanced MRI scanning. The scanned images were transmitted to the Varian Eclipse project system workstation, and the 2 images were fused to delineate the target volume.

The localized enhanced CT, MRI and CT and MRI fused images are shown in Figure 1A,B,C.

\section{Delineation and planning of the radiotherapy target volume}

Target volume delineation was determined as follows: the 
Table 1 Comparison of general data among the four groups

\begin{tabular}{|c|c|c|c|c|c|c|}
\hline Subjects & \multicolumn{4}{|c|}{ Groups } & $\chi^{2} / F$ value & $P$ value \\
\hline Sex & & & & & 0.576 & 0.633 \\
\hline Male & 12 & 13 & 11 & 9 & & \\
\hline Female & 8 & 7 & 9 & 11 & & \\
\hline $\mathrm{T}$ phase & & & & & 0.912 & 0.439 \\
\hline T1 & 1 & 3 & 2 & 3 & & \\
\hline T2 & 7 & 7 & 7 & 9 & & \\
\hline T3 & 12 & 10 & 11 & 8 & & \\
\hline N2 & 12 & 9 & 9 & 10 & & \\
\hline N3 & 6 & 8 & 9 & 7 & & \\
\hline Number of brain metastases & $6.05 \pm 1.395$ & $6.05 \pm 1.146$ & $6.00 \pm 1.026$ & $5.85 \pm 0.933$ & 0.138 & 0.937 \\
\hline
\end{tabular}

Single-factor analysis of count data of the 4 groups was performed with the $\chi^{2}$ test, while measurement data was analyzed using the $t$-test; $\mathrm{P}<0.05$ was significantly different.

whole brain was delineated as the clinical target volume (CTV), while the intracranial metastasis was delineated as the gross tumor volume (GTV); the CTV and GTV were enlarged to 5 and $10 \mathrm{~mm}$ and defined as the planning target volume PTV-CTV and PTV-GTV, respectively.

Radiotherapy plan formulation: four plans were developed for each patient, and the different parameters of different doses and segment mode radiotherapy plans were compared.

* Group A (WBRT plan 1): PTV-CTV prescription volume of $300 \mathrm{cGy} / 10$ fractions and a total of 3,000 cGy;

* Group B (WBRT alone plan 2): PTV-CTV prescription volume of $180 \mathrm{cGy} / 22$ factions, and a total of 3,960 cGy;

* Group C (radiotherapy plan for intracranial metastasis alone): PTV-GTV prescription volume of $250 \mathrm{cGy} / 22$ factions, and a total of 5,500 cGy;

- Group D (whole brain + intracranial metastasis radiotherapy plan): PTV-CTV prescription volume of $180 \mathrm{cGy} / 22$ fractions, a PTV-GTV the prescription volume of $250 \mathrm{cGy} / 22$ fractions, a total of 3,960 cGy, and a metastatic focus of $5,500 \mathrm{cGy}$ (calculated as $\mathrm{ds} / \mathrm{ds}=10$, with a biological equivalent of about $68 \mathrm{~Gy}$ ).

The specific radiotherapy plan was implemented according to the mode of patients randomized radiotherapy, and the efficacy and adverse reactions of radiotherapy were compared. In addition to group A, the other 3 groups were reset after 15 fractions of radiotherapy; the GTV was then redefined, and the radiotherapy plan was reformulated.

\section{Data analysis}

For each patient, 4 radiotherapy plans were developed and paired to compare different dose parameters, including the dose to the tumor and the organ at risk. The main parameters included: GTV, CTV, PTV, crystal, brainstem, optic nerve, and spinal cord. Differences in treatment outcomes among the 4 groups were compared, and intracranial progressionfree survival (IPFS) and OS were compared.

\section{Statistical analysis}

Statistical analysis was performed using SPSS software (version 17; SPSS, Inc., Chicago, IL, USA). Student's $t$-test was utilized to compare the means of four groups. The $\chi^{2}$ test was used for all other comparisons. Univariate survival 

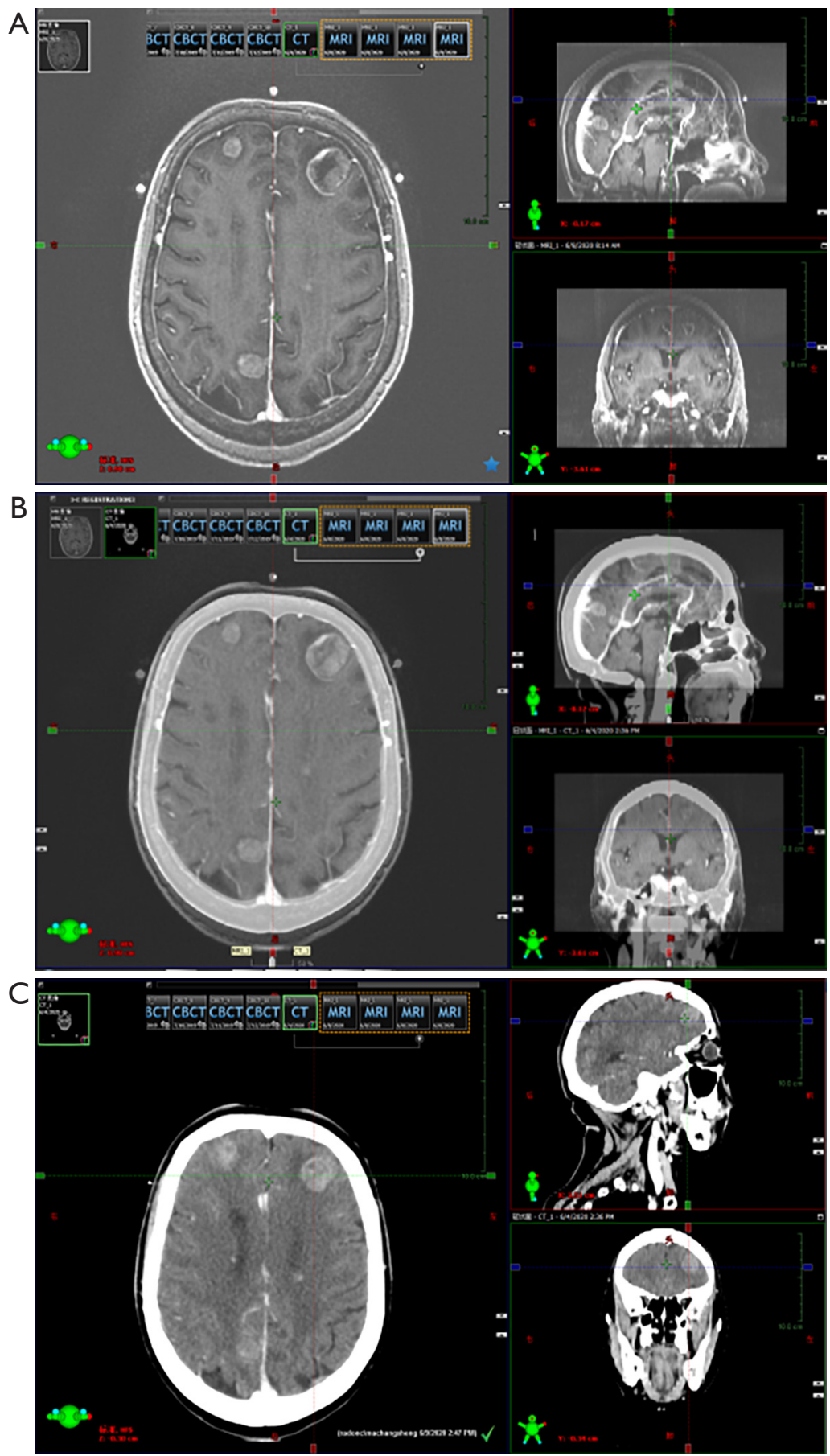

Figure 1 The localized enhanced CT, MRI and CT and MRI fused images. (A) The localized enhanced MRI image; (C) the localized enhanced CT image; (B) the localized enhanced CT and MRI fused images. CT, computed tomography. 
Table 2 Comparison of radiotherapy plan parameters in different doses and fractionated radiotherapy among the four groups

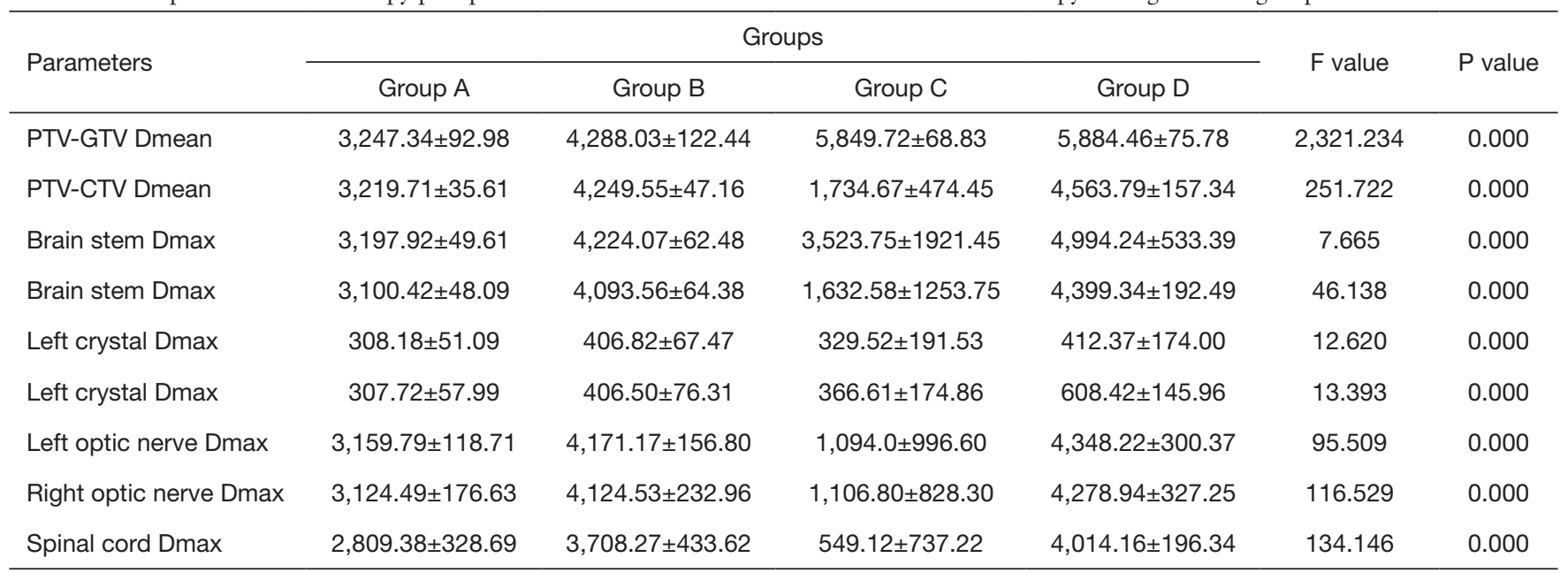

The dose unit is cGy, Dmax is the maximum dose, and Dmean is the average dose. PTV, planning target volume; GTV, gross tumor volume; CTV, clinical target volume.

analysis was conducted using Kaplan-Meier analysis and the log-rank test. The significance level was $\alpha=0.05$.

\section{Results}

Dose comparison of different parameters of different doses and fractionated radiotherapies

The dose of PTV-GTV and PTV-CTV, along with the dose of normal tissues such as crystals, brain stem, spinal cord etc. were statistically significant in the 4 groups $(\rho=0.000)$ (Table 2).

\section{Comparison of therapeutic effect of different doses and fractionated radiotherapies and multi-factor analysis of influencing IPFS}

In the comparison of the efficacy of radiotherapy with different doses and fractionated radiotherapys in the 4 groups, there was a significant difference in IPFS among the 4 groups, but no significant difference in OS (Table 3; Figures 2,3). The multi-factor analysis of influencing IPFS showed that the dose of WBRT, the dose of tumor radiotherapy, and the frequency of radiotherapy were the factors influencing IPFS (Table 4).

\section{Comparison of the adverse reactions of different doses and fractionated radiotherapies to the nervous system}

The poor status of the nervous system in patients of the 4 groups mainly manifested as memory decline, and there was a significant difference between the 4 groups in the comparison of memory decline at 6 months, 1 year, and 2 years after radiotherapy (Table 5).

\section{Discussion}

Radiotherapy is an effective treatment for brain metastasis, especially for multiple brain metastases patients. Currently, radiotherapy for multiple brain metastases is a WRBTbased radiation mode (4-6). Studies have reported that stereotactic radiotherapy or hypofractionated radiation therapy has better efficacy and less adverse reactions, and tends to be chosen over WBRT (7-10). However, for the intracranial control rate, the incidence of intracranial metastasis is relatively high (11-13). Therefore, WBRT is still the main treatment for multiple brain metastases.

WBRT dose is limited (generally less than 40-45 Gy), which can control the occurrence of intracranial transfer, but not for the existing intracranial metastatic site. Therefore, on the basis of WBRT, one of the clinically feasible radiotherapy methods is to add dose irradiation to the local metastatic tumor for the purposes of synchronous dose and intensity-modulated radiotherapy (14-16).

This prospective clinical controlled study found that radiotherapy with different doses and fractionated radiotherapy had significant differences in parameters, including the dose of metastatic tumor and the dose of normal tissue, with the dose of intracranial metastatic tumor 
Table 3 Comparison of median survival time (months) of IPFS and OS in the four groups

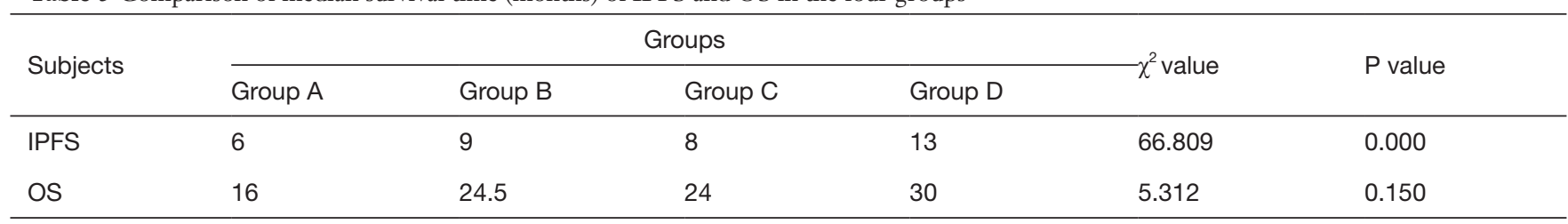

IPFS, intracranial progression-free survival; OS, overall survival.

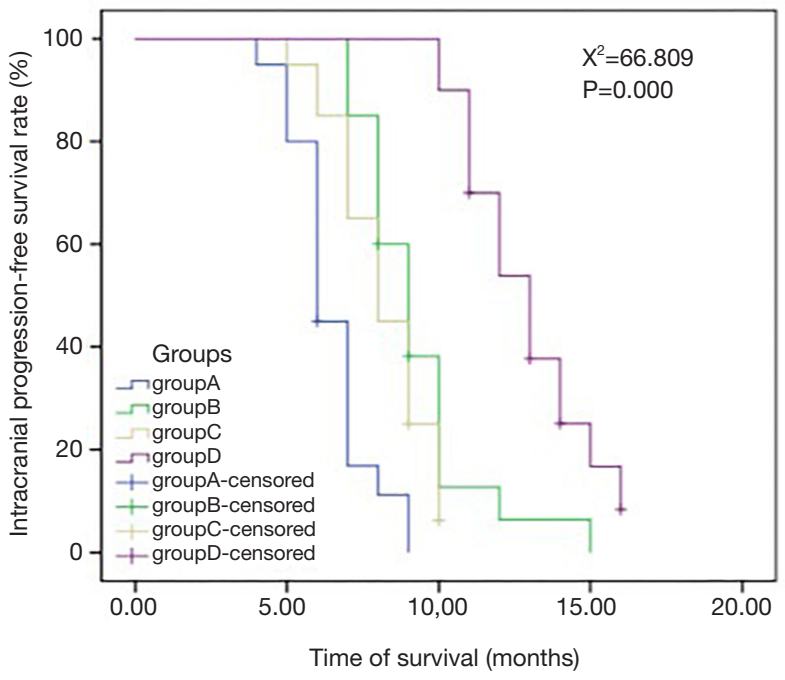

Figure 2 Intracranial progression-free survival curve of four groups of patients.

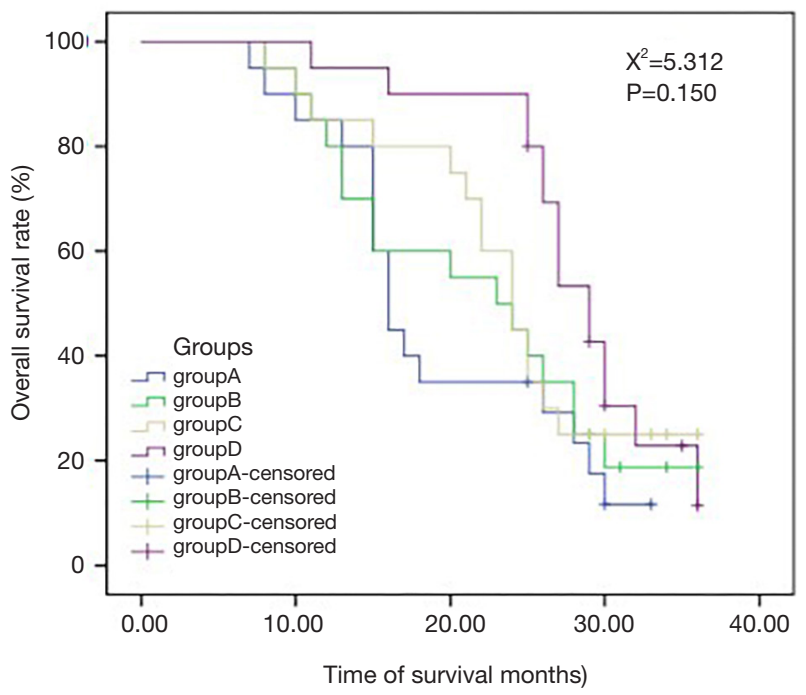

Figure 3 Overall survival curve of the four groups. being significantly different across the 4 groups $(\mathrm{P}=0.000)$. Different segmentation patterns and doses were used to prevent intracranial metastatic tumors and the whole brain as well, leading to the differences in local tumor control rates and IPFS survival. The doses from normal tissues and organs, such as bilateral crystals, the bilateral optic nerve, brainstem and spinal cord, were significantly different across the 4 groups $(\mathrm{P}=0.000)$. Therefore, with the increase of the radiotherapy dose of the whole brain and intracranial metastatic site, the dose of organs at risk also increases correspondingly, but within the tolerable range of organs at risk.

In the comparison of the 4 groups in terms of different doses and fractionated radiotherapy effects, the IPFS of the 4 groups of patients respectively was $6,9,8$, and 13 months $\left(\mathrm{X}^{2}=66.809, \mathrm{P}=0.000\right)$, revealing a significant difference. The median OS of the 4 groups of patients (OS) was 16 , 24.5, 24, and 30 months, respectively $\left(\mathrm{X}^{2}=5.312, \mathrm{P}=0.150\right)$, but there was no significant difference between 4 groups. WBRT in the intracranial metastasis group (SIB) (group D) showed a tendency of increased OS, but a larger sample size is needed to adequately confirm this. Studies have reported that WBRT improves local control rate but not the OS, which is similar to the results reported in the literature $(2,13,17-20)$. The reasons for this may be as follows. Firstly, lung adenocarcinoma patients with brain metastases are often accompanied by metastases to other tissues or organs. Secondly, isolated metastatic sites or oligo-metastases can be cured, but for multiple metastatic sites, especially for multiple brain metastases, the radical radiotherapy dose cannot be reached because of the influence of the normal tolerated dose of organs at risk. Third, after the first course of chemoradiotherapy, the general condition of the patients decreases due to the adverse reactions of the treatment, and the patients are unable to tolerate chemoradiotherapy and other treatments, leading to the progression of the disease. Fourth, elderly patients with chronic complications such as coronary heart disease, diabetes, chronic lung disease, etc., cannot tolerate anti-tumor treatment; fifth, patients may 
Table 4 Cox multivariate analysis of IPF

\begin{tabular}{lccccc}
\hline Factors & $\beta$ & SE & Wald value & RR & $95 \% \mathrm{Cl}$ \\
\hline Dose of WBRT & -0.123 & 0.034 & 13.261 & 0.884 & $0.828-0.945$ \\
Dose of tumor & -0.242 & 0.052 & 21.491 & 0.785 & $0.708-0.869$ \\
Fractions & 0.175 & 0.069 & 6.457 & 1.191 & $1.041-1.364$ \\
\hline
\end{tabular}

IPF, intracranial progression-free; WBRT, whole brain radiotherapy.

Table 5 Comparison of adverse nerve system reactions among the four groups

\begin{tabular}{|c|c|c|c|c|c|c|}
\hline Time & \multicolumn{4}{|c|}{ Group } & $\chi^{2}$ value & $P$ value \\
\hline Six months & $2,10.0$ & $3,15.0$ & $1,5.0$ & $3,15.0$ & 18.000 & 0.006 \\
\hline One year & $4,20.0$ & $6,30.0$ & $2,10.0$ & $7,35.0$ & 57.000 & 0.000 \\
\hline Two years & $9,45.0$ & $12,60.0$ & $4,20.0$ & $13,65.0$ & 126.000 & 0.000 \\
\hline
\end{tabular}

The time was calculated after radiotherapy.

not be able to continue treatment due to economic reasons. Thus, after the disease has stabilized for a while, the patient's disease may progress slowly or rapidly, leading to death. SRS combined with WRBT has also been reported to improve survival over SRS (21).

The results of multi-factor analysis showed that multifactors influencing IPFS included WRBT dose, radiotherapy dose for tumor, and radiotherapy times, among WRBT dose and radiotherapy dose for tumor were protective factors and related to the survival of patients. The higher the dose of radiotherapy is for the whole brain and intracranial metastatic tumor, the better the effect on IPFS.

Adverse effects on the nervous system among the 4 groups of patients mainly manifested as memory decline at 6 months, 1 year, and 2 years after radiation treatment. In the comparison of the 4 groups' memory decline, it was found that as time went by, the incidence of nervous system adverse effects rose year by year, with the 4 groups having significant differences, and the WBRT and WRBT dose being the main factors affecting the nerve system function. A study reported that WRBT protects the hippocampus and can reduce the occurrence of nervous system adverse effects (22-24). As there is a low incidence of brain metastasis in the intracranial hippocampus and hippocampus, hippocampal protection is possible (25-27). On the basis of hippocampal protection, WRBT combined with intracranial metastasis SIB will be the focus and direction of future clinical research.

\section{Conclusions}

WBRT is one of the main treatment methods for multiple brain metastases. Simultaneous dosing on the basis of WRBT can improve the local control rate and potentially improve OS. Therefore, WRBT combined with intracranial metastasis plus SIB has potential as the standard mode of radiotherapy for multiple brain metastases. Adverse effects of the nerve system are related to the dose of WRBT and the time after radiotherapy. How to protect the hippocampus and reduce the adverse reactions to the nervous system while performing WRBT should be the focus of future research.

\section{Acknowledgments}

Funding: This work was financially supported by the Key Research and Development Project of Shandong Province (2015GSF118027), and the National Natural Science Foundation of China (81974467).

\section{Footnote}

Reporting Checklist: The authors have completed the CONSORT reporting checklist. Available at http://dx.doi. org/10.21037/apm-20-1203

Data Sharing Statement: Available at http://dx.doi. 
org/10.21037/apm-20-1203

Conflicts of Interest: All authors have completed the ICMJE uniform disclosure form (available at http://dx.doi. org/10.21037/apm-20-1203). The authors have no conflicts of interest to declare.

Ethical Statement: The authors are accountable for all aspects of the work in ensuring that questions related to the accuracy or integrity of any part of the work are appropriately investigated and resolved. The study was conducted in accordance with the Declaration of Helsinki (as revised in 2013). Approval was obtained from the ethics committee of our hospital (No. SDTHEC201503048), and the patients signed the informed consent and were followed up regularly as required.

Open Access Statement: This is an Open Access article distributed in accordance with the Creative Commons Attribution-NonCommercial-NoDerivs 4.0 International License (CC BY-NC-ND 4.0), which permits the noncommercial replication and distribution of the article with the strict proviso that no changes or edits are made and the original work is properly cited (including links to both the formal publication through the relevant DOI and the license). See: https://creativecommons.org/licenses/by-nc-nd/4.0/.

\section{References}

1. Fidler IJ. The Biology of Brain Metastasis: Challenges for Therapy. Cancer J 2015;21:284-93.

2. Owonikoko TK, Arbiser J, Zelnak A, et al. Current approaches to the treatment of metastatic brain tumours. Nat Rev Clin Oncol 2014;11:203-22.

3. Gong X, Zhou D, Liang S, Analyses of prognostic factors in cases of non-small cell lung cancer with multiple brain metastases. Onco Targets Ther 2016;9:977-83.

4. Mizuno T, Takada K, Hasegawa T, et al. Comparison between stereotactic radiosurgery and whole-brain radiotherapy for 10-20 brain metastases from non-small cell lung cancer. Mol Clin Oncol 2019;10:560-6.

5. Buss EJ, Wang TJ. Treatment of lung adenocarcinoma brain metastases: what is the role of radiotherapy in the age of precision medicine? Transl Lung Cancer Res 2018;7:S318-20.

6. Martinive P, Van Houtte P. The challenge of brain metastases from non-small cell lung cancer is not only an economical issue. Ann Palliat Med 2019;8:203-6.
7. Jiang $W$, Haque $W$, Verma $V$, et al. Stereotactic radiosurgery for brain metastases from newly diagnosed small cell lung cancer: practice patterns and outcomes. Acta Oncol 2019;58:491-8.

8. Bowden G, Faramand A, Niranjan A, et al. Gamma Knife Radiosurgery for the Management of More Than 15 Cerebral Metastases. World Neurosurg 2019;126:e989.

9. Zairi F, Ouammou Y, Le Rhun E, et al. Relevance of gamma knife radiosurgery alone for the treatment of non-small cell lung cancer brain metastases. Clin Neurol Neurosurg 2014;125:87-93.

10. Tamari K, Suzuki O, Hashimoto N, et al. Treatment outcomes using CyberKnife for brain metastases from lung cancer. J Radiat Res 2015;56:151-8.

11. Gu X, Zhao YQ, Xu F. Whole Brain Irradiation and Hypo-fractionation Radiotherapy for the Metastases in Non-small Cell Lung Cancer. Chinese Journal of Lung Cancer 2016;19:224-9.

12. Aoyama H, Shirato H, Tago M, et al. Stereotactic radiosurgery plus whole-brain radiation therapy vs stereotactic radiosurgery alone for treatment of brain metastases. A randomized controlled trial. JAMA 2006;295:2483-91.

13. Yamamoto M, Serizawa T, Shuto T, et al. Stereotactic radiosurgery for patients with multiple brain metastases (JLGK0901): a multi-institutional prospective observational study. Lancet Oncol 2014;15:387-95.

14. Cheng XB, Xiao L. Feasibility study on the treatment for brain metastases of lung cancer via intensity-modulated radiotherapy combined with simultaneous integrated boost. Chin Oncol 2016;26:687-92.

15. Qin XL, Yu ZL, Yang F. The optimization of radiotherapy for the treatment of intracranial metastases. Journal of Modern Oncology 2015;1:45-8.

16. Aoyama H, Tago M, Shirato H, et al. Stereotactic Radiosurgery With or Without Whole-Brain Radiotherapy for Brain Metastases: Secondary Analysis of the JROSG 99-1 Randomized Clinical Trial. JAMA Oncol 2015;1:457-64.

17. Brown PD, Jaeckle K, Ballman KV, et al. Effect of Radiosurgery Alone vs Radiosurgery With Whole Brain Radiation Therapy on Cognitive Function in Patients With 1 to 3 Brain Metastases: A Randomized Clinical Trial. JAMA 2016;316:401-9.

18. Chang EL, Wefel JS, Hess KR, et al. Neurocognition in patients with brain metastases treated with radiosurgery or radiosurgery plus whole-brain irradiation: a randomised controlled trial. Lancet Oncol 2009;10:1037-44. 
19. Feng Y, Hu XS. Progress in the treatment of non-small cell lung cancer with brain metastases. Chinese Journal of Clinical Oncology 2018;(7):331-8.

20. Gong X, Zhou D, Liang S, Analyses of prognostic factors in cases of non-small cell lung cancer with multiple brain metastases. Onco Targets Ther 2016;9:977-83.

21. Aoyama H, Tago M, Shirato H, et al. Stereotactic Radiosurgery With or Without Whole-Brain Radiotherapy for Brain Metastases: Secondary Analysis of the JROSG 99-1 Randomized Clinical Trial. JAMA Oncol 2015;1:457-64.

22. Han YM, Chai WM, Xu C, et al. Radiological features of non-small-cell lung cancer with brain metastases in a single center: an implication for the HA-WBRT approach. Chinese Journal of Radiation Oncology 2018;27:798-804.

23. Gondi V, Pugh SL, Tome W A, et al. Preservation of Memory With Conformal Avoidance of the Hippocampal Neural Stem-Cell Compartment During Whole-Brain Radiotherapy for Brain Metastases (RTOG 0933): A Phase
II Multi-Institutional Trial. J Clin Oncol 2014;32:3810-6.

24. Dong X, Zhou ZM, Liu X, et al. Phase II clinical trial of hippocampal-sparing prophylactic cranial irradiation in patients with small cell lung cancer. Chinese Journal of Radiation Oncology 2018;27:638-42.

25. Marsh JC, Herskovic AM, Gielda BT, et al. Intracranial Metastatic Disease Spares the Limbic Circuit: A Review of 697 Metastatic Lesions in 107 Patients.Int J Radiat Oncol Biol Phys 2010;76:504-12.

26. Gondi V, Tome WA, Marsh J, et al. Estimated risk of perihippocampal disease progression after hippocampal avoidance during whole-brain radiotherapy: safety profile for RTOG 0933. Radiother Oncol 2010;95:327-31.

27. Zhao L, Shen Y, Guo JD, et al. Analyses of distribution and dosimetry of brain metastases in small cell lung cancer with relation to the neural stem cell regions: feasibility of sparing the hippocampus in prophylactic cranial irradiation. Radiat Oncol 2017;12:118-219.
Cite this article as: Liu $\mathrm{H}, \mathrm{Xu} \mathrm{X}$, Wang J, Wang W, Ma C, Sun T, Shao Q. Clinical study on different doses and fractionated radiotherapies for multiple brain metastases of non-EGFR mutant lung adenocarcinoma. Ann Palliat Med 2020;9(4):2003-2012. doi: 10.21037/apm-20-1203 